\title{
A Clinical Study to Compare the Efficacy of Pretreatment with Granisetron and Lignocaine to Alleviate Pain on Propofol Injection
}

\author{
B. P. Manjula ${ }^{1}$, Balachandra S. R. ${ }^{2}$ \\ ${ }^{1}$ Professor Department of Anaesthesiology, J.S.S Medical College and University, Mysore \\ ${ }^{2}$ Junior Resident, Department of Anaesthesiology, J.S.S Medical College and University, Mysore
}

\begin{abstract}
Pain on injection of anaesthetic is an important cause of patient dissatisfaction and a recognized adverse effect of propofol. Routinely, intravenous preservative free lignocaine is used for prevention of pain due to injection of propofol. In this study, granisetron a specific $5 \mathrm{HT}_{3}$ receptor antagonist was used to compare the efficacy. 100 adult patients belonging to ASA physical status I or II, scheduled for elective surgeries under general anaesthesia were randomly allocated into two groups $A$ \& B. Group A received Inj lignocaine $30 \mathrm{mg}$ and group B received Inj granisetron $2 \mathrm{mg}$ as pretreatment, before injection of propofol. Comparing pain during propofol injection, lignocaine and granisetron were found to decrease the injection pain significantly. Granisetron has less incidence of PONV.
\end{abstract}

Keywords: Granisetron, lignocaine, pain on injection, propofol, PONV

\section{Introduction}

"The relief of pain is purchased always at a price. The price in both morbidity and mortality does not greatly differ whatever the agent or agents used." - R.M.Waters with a decrease in morbid adverse events after surgery, patient satisfaction with peri-operative care is assuming more importance. Pain on injection of anaesthetic is an important cause of patient dissatisfaction and is a recognised adverse effect of propofol.[1]

The reported incidence of pain on injection of propofol varies between $28 \%$ and $90 \%$ in adults if a vein on dorsum of the hand is used.[2] It is known to cause severe, sharp, stinging or burning pain on injection that can be distressing to the patient. This pain is considered to be clinically unacceptable as it can cause agitation and interfere with smooth induction of anaesthesia.

Many pharmacological \& non pharmacological methods have been tried to alleviate pain on propofol injection with variable success results

Intravenous lignocaine, a local anaesthetic, has been well documented to reduce the incidence and severity of pain on injection of propofol.[3][4][5] It is considered superior to other drugs but cannot reduce the incidence and severity of pain on intravenous injection of propofol completely. Studies concluded that $30 \mathrm{mg}$ of lidocaine is optimal for reducing pain on propofol injection and there was no improvement when dose was increased.

Granisetron, a specific $5 \mathrm{HT}_{3}$ receptor antagonist exhibits properties of a local anaesthetic: provided numbness when injected under skin. It has been demonstrated that Granisetron was effective in alleviating pain following propofol injection without any adverse effects. Granisetron had been shown to relieve pain by its multi-faceted actions.

So we undertaken a study in hundred patients of ASA physical status I and II, age ranging between 18 to 50years undergoing elective surgeries under general anaesthesia who were randomly allocated into two groups of 50 each. In this study we compare the efficacy of intravenous granisetron with intravenous lignocaine to alleviate pain on propofol injection during general anaesthesia and incidence of post operative nausea and vomiting within 24 hours.[15]

\section{Literature Survey}

Studies conducted by O. Canbayet al on Efficacy of intravenous acetaminophen and lidocaine on propofol injection pain,[6] Sharon.Y.King et al on Lidocaine for the prevention of pain due to propofol,[5] In a study conducted by Ahsan Ahmed et al on Pretreatment with intravenous granisetron to alleviate pain on propofol injection, $2 \mathrm{mg}$ of granisetron was used and concluded that it was effective in preventing pain on propofol injection.[1]

\section{Methodology}

Institutional ethical committee approval was obtained and informed written consent was taken from all the patients.

ASA I or II, aged between 18 and 50 years presenting for elective surgery under general anaesthesia were included in the study.

Patients with difficulty in communication, Patients with a history of allergic response to propofol or $5 \mathrm{HT}_{3}$ receptor antagonist or lignocaine \& Pregnant women were excluded from the study. 


\section{International Journal of Science and Research (IJSR) \\ ISSN (Online): 2319-7064}

Index Copernicus Value (2013): 6.14 | Impact Factor (2014): 5.611

Patients were explained about the procedure during the preanaesthetic visit. Hundred patients were randomly allocated to two different groups of 50 each as described below (group $\mathrm{A}$ and $\mathrm{B})$.

On arrival in the operation room, a 20G cannula was inserted into a vein on the dorsum of patient's non dominant hand and started on Ringers lactate solution. Monitors were connected, heart rate, non-invasive blood pressure, peripheral $\mathrm{O}_{2}$ saturation and respiratory rate were recorded before injecting propofol and at 1 and 3 minute after propofol injection.

In our study, we used a non- pneumatic tourniquet which was maintained for one minute duration during pretreatment and released prior to propofol injection.

Patients in GROUP A received 1\% (30mg) lignocaine and GROUP B received $2 \mathrm{ml}$ of $2 \mathrm{mg}$ granisetron, over a period of 5 seconds.

In this study we avoided any kind of IV premedication (other than the study drugs) which may cause irritation or analgesia before injection of propofol.

Patients were induced with propofol $2 \mathrm{mg} / \mathrm{kg}$. Initially $2 \mathrm{ml}$ bolus of propofol was injected over 4 seconds, 15 seconds later patient was asked to rate any sensation of pain during injection of propofol.

The grading of pain was done using Mc Crirrick and Hunter scale of evaluation of propofol injection. An anaesthesiologist blinded to study protocol evaluated pain during propofol injection using above mentioned scale (Mc Crirrick and Hunter scale):

Table 1: Mc Crirrick and Hunter Scale of Evaluation of Propofol Injection Pain

\begin{tabular}{|c|c|}
\hline 0 & none (negative response to questioning) \\
\hline 1 & $\begin{array}{c}\text { mild pain (pain reported only in response to questioning } \\
\text { without any behavioural signs) }\end{array}$ \\
\hline 2 & $\begin{array}{c}\text { moderate pain (pain reported in response to questioning } \\
\text { and accompanied by a behavioural sign or pain reported } \\
\text { spontaneously without questioning) }\end{array}$ \\
\hline 3 & $\begin{array}{c}\text { severe pain (strong vocal response or response } \\
\text { accompanied by facial grimacing, arm withdrawal or } \\
\text { tears) }\end{array}$ \\
\hline
\end{tabular}

Followed with induction \& intubation with appropriate tube size. Anaesthesia was maintained using $\mathrm{O} 2$ in $\mathrm{N} 2 \mathrm{O}$ and $0.5 \%$ halothane uniformly in all cases. For muscle relaxation Inj.Vecuronium bromide $0.1 \mathrm{mg} \mathrm{kg-1}$ was given as loading dose and one fourth of loading dose was used for maintenance.

Patients were reversed with Inj.Neostigmine $0.05 \mathrm{mg} \mathrm{kg}-1$ and Inj. Glycopyrrolate $0.01 \mathrm{mg} \mathrm{kg-1}$ both IV after ensuring adequate recovery from neuromuscular blockade. Oral cavity and throat were suctioned thoroughly prior to extubation, and extubated once extubation criteria was met.

\section{Statistical Analysis}

Data was analyzed using Chi square test, unpaired t test, descriptive statistics and frequencies. The results are presented as mean $+/$ - standard deviation. For all the tests $\mathrm{P}$ value of $\leq 0.05$ is considered as statistically significant.

\section{Results}

Data was collected and statistical analysis was performed as explained in the methodology of the study. The results and interpretations are as explained below.

\subsection{Age Distribution in Study Groups}

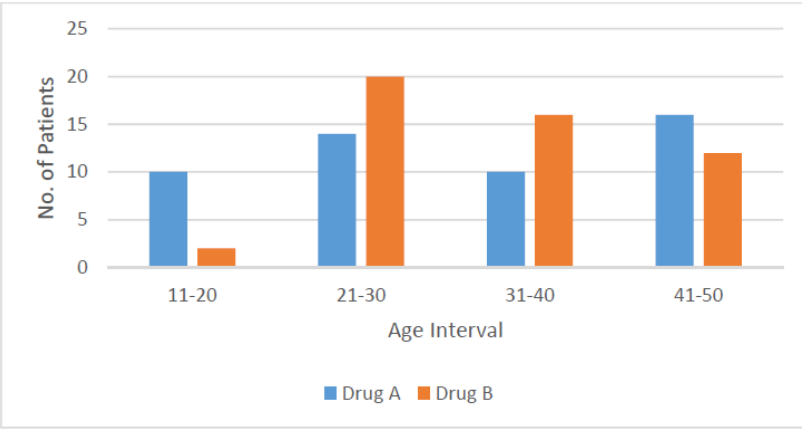

Graph 1: Age Distribution

Contingency Coefficient $=0.278, \mathrm{P}$ value $=0.039$

In both groups Drug A and Drug B, the age distribution ranged from $18-50$ years. Maximum number of patients in 41-50 years (32\%) in Drug A and 21-30 years (40\%) in Drug B.

\subsection{Comparison of Changes in Heart Rate in Study Groups:}

Table 2: Heart Rate Variability

\begin{tabular}{|c|c|c|c|c|}
\hline \multirow{2}{*}{$\begin{array}{c}\text { Heart rate } \\
\text { Beats per min }\end{array}$} & \multicolumn{4}{|c|}{ Groups } \\
\cline { 2 - 5 } & \multicolumn{2}{|c|}{ Drug A } & \multicolumn{2}{c|}{ Drug B } \\
\cline { 2 - 5 } & 1 min & $3 \mathrm{~min}$ & $1 \mathrm{~min}$ & $3 \mathrm{~min}$ \\
\hline $0-10$ & 43 & 32 & 43 & 34 \\
\hline $11-20$ & 6 & 11 & 4 & 13 \\
\hline $21-30$ & 1 & 6 & 2 & 3 \\
\hline $31-40$ & 0 & 1 & 1 & 0 \\
\hline
\end{tabular}

The heart rate both groups were compared before induction, during induction intraoperatively at $1 \mathrm{Min}, 3 \mathrm{Min}$ periods and it is not statistically significant ( $P$ value $=0.822$ ). The maximum variability in heart rate is seen in $1^{\text {st }}$ minute after induction in both groups.

\subsection{Comparison of Changes in Systolic \& Diastolic Blood Pressure in Study Groups}




\section{International Journal of Science and Research (IJSR) \\ ISSN (Online): 2319-7064}

Index Copernicus Value (2013): 6.14 | Impact Factor (2014): 5.611

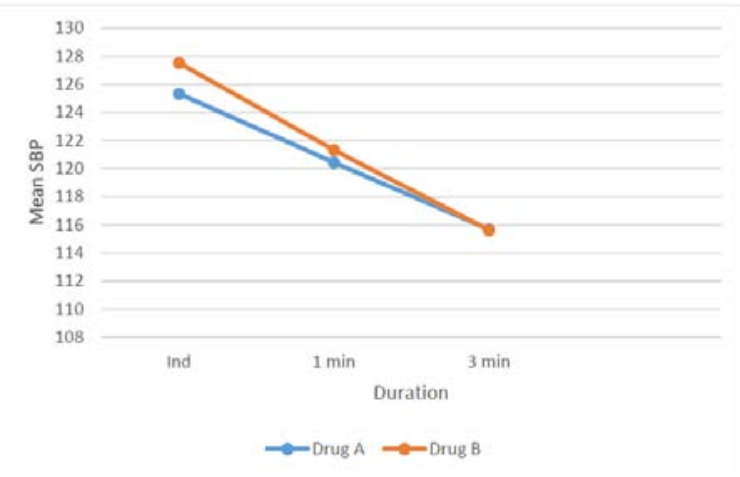

Graph 2 : Changes in Systolic Blood Pressure in Two Groups

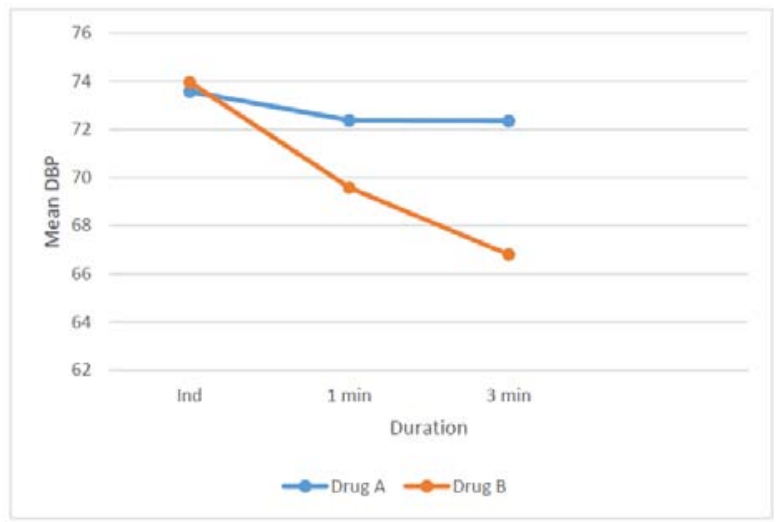

Graph 3: Changes in Diastolic Blood Pressure in Two Groups.

The systolic and diastolic blood pressure in both groups was compared before induction, during induction intra operatively at $1 \mathrm{~min}, 3 \mathrm{~min}$ period and it is not statistically significant.

\subsection{Comparison of Mean SPO2 in Study Groups}

$\mathrm{SPO}_{2}$ in both groups were compared before induction, during induction intraoperatively at $1 \mathrm{~min}, 3 \mathrm{~min}$ period and is not statistically significant

\subsection{Comparison of Pain During Induction in Study Groups}

Comparing pain during propofol injection, $76.0 \%$ in Drug A group and $62.0 \%$ in Drug B group did not have pain, $12.0 \%$ in Drug A group and $20.0 \%$ in Drug B group had mild pain, $12.0 \%$ in Drug A group and $18.0 \%$ in Drug B group had Moderate pain, and $0.0 \%$ in both the groups had severe pain. But difference between two groups is statistically insignificant.

\subsection{Comparison of PONV in Study Groups}

In our study, 13 patients in group A and 4 patients in group B complained of nausea and vomiting during $24 \mathrm{hr}$ postoperative period.

\section{Discussion}

The most worrying side effect of propofol which has been most extensively studied is pain on injection of propofol. The incidence of pain varies from $28-90 \%$ of patients and in children $28-85 \%$.[5] . Early researchers quoted that in spite of attractive properties of this drug, the high incidence of pain on injection will make it take a back seat in due course of time.

Although the underlying mechanisms are still not fully understood,[7], the explanation for the pain include endothelial irritation, osmolality differences, unphysiological $\mathrm{pH}$ and the activation of pain mediators. [8]

The initial component of pain, involving immediate stimulation of nociceptors and free nerve endings [7] seems to be associated mainly with the concentration of free drug within the aqueous phase of the emulsion.[11] The delayed component of pain, appearing within half a minute, is also believed to result from interaction with nociceptors and free nerve endings [7]; however, promoted by local vasodilatation and hyperpermeability induced by bradykinin [12], [13] and possibly also prostaglandin E2. [10], [12].

Though many methods of alleviating or reducing the intensity of pain on injection of propofol have been studied, the most commonly drug used for pretreatment was lignocaine. Different concentrations and doses of lignocaine were tried to find optimal dosage of lignocaine. Addition of $1 \%$ lignocaine to $1 \%$ Propofol $(1: 10)$ caused propofol to migrate from the aqueous phase of emulsion into its lipid phase. Studies concluded that $30 \mathrm{mg}$ of lidocaine is optimal for reducing pain on propofol injection and there was no improvement when dose was increased.

Granisetron a specific $5 \mathrm{HT}_{3}$ receptor antagonist was used to compare with lignocaine to alleviate pain on propofol injection.

Granisetron had been shown to relieve pain by its multifaceted actions as a

- $\mathrm{Na}+$ channel \& $\mathrm{K}+$ channels Block: Blocks the fast $\mathrm{Na}+$ \& $\mathrm{K}+$ channels locally and hence transmission and modulation of neuronal response in Peripheral and Central Nervous system.

- Acts as local anaesthetic.[14].

- $5 \mathrm{HT}_{3}$ antagonist: Antagonism of peripheral $5 \mathrm{HT}_{3}$ receptors involving nociceptive pathway.[14]

- Mu opioid agonist: Granisetron bind to mu receptors present peripherally and in spinal cord \& acts as agonist leading to analgesic effect.[14]

The dose of granisetron used in our study was $2 \mathrm{ml}(1 \mathrm{mg} / \mathrm{ml})$ as this dose was considered to be effective as in previous study.[1]

In our study, distribution of age ranged between 18-50 yrs with the mean age of lignocaine group being 38yrs and for granisetron group being 40 yrs. Majority of patients in both 


\section{International Journal of Science and Research (IJSR) \\ ISSN (Online): 2319-7064}

Index Copernicus Value (2013): 6.14 | Impact Factor (2014): 5.611

groups were males, $64 \%$ in lignocaine group and $52 \%$ in granisetron group.

The sex difference between the groups is statistically insignificant. The distribution of weight and ASA physical status between the groups are statistically insignificant. Hence demographic characteristics are similar and comparable in both groups.

Changes in the parameters showed similar pattern in both groups and are statistically insignificant. Thus both lignocaine and granisetron did not cause any significant hemodynamic disturbances in our study. Even in patients who experienced pain on propofol injection, increase in heart rate was not significant enough to cause hemodynamic instability.

Out of 50 patients of each study group, $76 \%$ in lignocaine group and $62 \%$ in granisetron group did not have pain, $12 \%$ in lignocaine group and $20 \%$ in granisetron group had mild pain, $12 \%$ in lignocaine \& $18 \%$ in granisetron group had moderate pain, severe pain was absent in both groups.

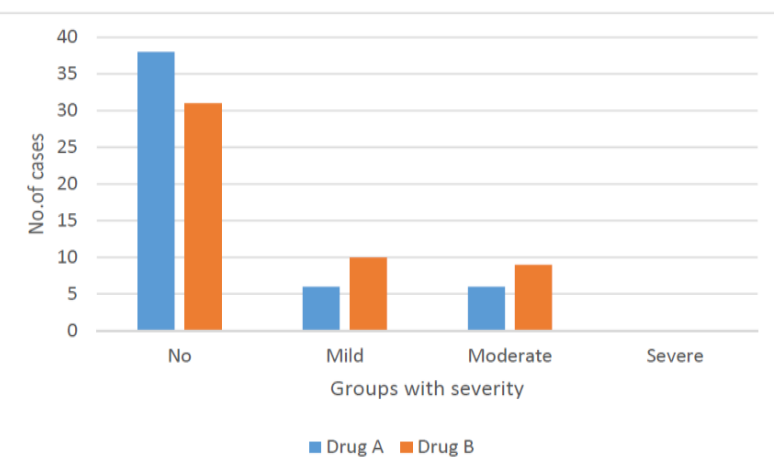

Graph 4: Comparison of Pain During Induction With Propofol

Contingency Co-efficient $=0.150, \mathrm{P}$ value $=0.315$

Thus both granisetron $2 \mathrm{mg}$ and lignocaine $30 \mathrm{mg}$ significantly reduced pain on propofol injection but there was no statistical significance between the two groups.

This is comparable to study conducted by Ahmed et al in 2012.[1] We observed 76\% of patients did not experienced pain on propofol which is comparable to study by Michael $\mathrm{H}$. Nathanson which concluded $87 \%$ of patients did not experienced pain.[3]

In our study, 13 patients $(26 \%)$ in group $\mathrm{A}$ and 4 patients $(8 \%)$ in group B complained of nausea and vomiting during $24 \mathrm{hr}$ postoperative period. It is comparable to incidence of 5$10 \%$ of PONV in a study Prophylactic Antiemetic Therapy with Ondansetron, Granisetron and Metoclopramide in Patients Undergoing Laparoscopic Cholecystectomy Under GA conducted by Vishal Gupta.[15]

Table 3: Comparision of PONV In Two Groups

\begin{tabular}{|l|l|l|}
\hline Groups & Number of patients & Percentage \\
\hline Drug A & 13 & $26 \%$ \\
\hline Drug B & 4 & $8 \%$ \\
\hline
\end{tabular}


[13] Ohmizo H, Obara S, Iwama H. Mechanism of injection pain with long and long-medium chain triglyceride emulsive propofol. Can J Anaesth 2005;52:595-9.

[14] Swati Bhatt, Bipin Kumar Pathak and M.R. Upadhyay. Granisetron - a novel drug for pain alleviation in propofol injection. World journal of pharmacy and pharmaceutical sciences. 2015 Volume 4, Issue 07, 1686-1701.

[15] Gupta V, Wakhloo R, Mehta A, Gupta SD. Prophylactic Antiemetic Therapy with Ondansetron, Granisetron and Metoclopramide in Patients Undergoing Laparoscopic Cholecystectomy Under GA.jk.science.org; 2008;10(2):74-77.

\section{Author Profile}

Dr. B.P. Manjula M.D. is currently working as Professor in Anaesthesiology in JSS Mediacal College and Hospital, Mysuru, Karnataka, India. She has been a resource person for various conferences and also has published research papers in national and international journals.

Dr. Balachandra S. R. received MBBS degree from JJM Medical College, Davanagere, India, he is working as junior resident in the department of Anaesthesiology in JSS medical college and hospital, Mysuru, India 